\title{
Valley properties of doped graphene in a magnetic field
}

\author{
J.S. Ardenghi ${ }^{\dagger}$, P. Bechthold ${ }^{\dagger}$, E. Gonzalez ${ }^{\dagger}$, P. Jasen ${ }^{\dagger}$ and A. Juan ${ }^{\dagger}$ \\ ${ }^{\dagger}$ IFISUR, Departamento de Física (UNS-CONICET) \\ Avenida Alem 1253, Bahía Blanca, Buenos Aires, Argentina
}

March 5, 2018

\begin{abstract}
The aim of this work is to describe the electronic properties of graphene in a constant magnetic field in the long wavelength approximation with random binary disorder, by solving the Soven equation self-consistently. Density of state contributions for different valleys in each sublattice sites are obtained for different values of magnetic field strength showing remarkable differences between $K$ and $K^{\prime}$ valleys. A band gap is obtained by an asymmetric on-site impurity concentration and the graphene electrons acquire an anomalous magnetic moment, which is opposite in different valleys, which depend highly in the interplay between the impurity band, the band edges and the broadening of the Landau levels. In turn, magnetization as a function of $B$ for different on-site random impurities is computed showing that by decreasing the on-site impurity energy values, maximum magnetization is shifted towards higher values of $B$ which can be used to create and manipulate polarized valley currents. Finally, conductivity and local vertex function are obtained as a function of energy showing that scattering contributions from $A$ and $B$ sublattices differ significantly. Effective medium local two-irreducible vertex is computed showing that scattering from sublattice $A$ and $B$ do not contribute equally, which can be related to weak anti-localization. From these results, it could be possible to explore how the valley pseudospin can be used to create polarized currents by populating asymmetrically the sublattice sites, where the population can be tuned with the applied magnetic field strength.
\end{abstract}

\section{Introduction}

Graphene is a two-dimensional allotrope of carbon which has become one of the most significant topics in solid state physics due to the large number of applications $([1,[2,3,4,4,5])$. The carbon atoms form a honey-comb lattice made of two interpenetrating triangular sublattices, $A$ and $B$. A special feature of the graphene band structure is the linear dispersion at the Dirac points which are dictated by the $\pi$ and $\pi^{\prime}$ bands that form conical valleys touching at the two independent high symmetry points at the corner of the Brillouin zone, the so called valley pseudospin [6. In the absence of defects, electrons near these symmetry points behave as massless relativistic Dirac fermions with an effective Dirac-Weyl Hamiltonian 44. When a magnetic field is applied perpendicular to the graphene sheet, a discretization of the energy levels is obtained, the so called Landau levels [7. These quantized energy levels still appear also for relativistic electrons, just their dependence on field and quantization parameter is different. In a conventional non-relativistic electron gas, Landau quantization produces equidistant energy levels, which is due to the parabolic dispersion law of free electrons. In graphene, the electrons have relativistic dispersion law, which strongly modifies the Landau quantization of the energy and the position of the levels. In particular, these levels are not equidistant as occurs in a conventional non-relativistic electron gas in a magnetic field. This large gap allows one to observe the quantum Hall effect in graphene, even at room temperature [8]. In turn, the valley pseudospin can

\footnotetext{
*email: jsardenghi@gmail.com, fax number: +54-291-4595142
} 
be used to create polarized currents in a similar way as the real electron spin is used in spintronics. By applying a local gate voltage to a quantum nano-contact in a graphene nanoribbon, valley filter can be obtained. Due to the finite size of the graphene sheet, the transvesal momentum component are quantized. Those states with a definite group velocity, depending on the polarized gate voltage sign applied, can go through the quantum nano-contact with the result of a largest population in one of the valleys. Altough the theoretical approach to distinguish carriers in the two valleys are known, the experimental procedure has become an attracting literature (see [9], [10, 11] and [12]). This valley pseudopin carries real magnetic moment, like real spin, supported by chiral orbital current, which can rise Zeeman splitting and Pauli paramagnetism in graphene [13. This magnetic moment can be used to couple it with a perpendicular magnetic field, which can be about 30 times the Bohr magneton for low energy electrons [9. However, taking into account the total contributions of the valley pseudospin, there is no net polarization by the magnetic field because the contribution of the Zeeman term to the Hamiltonian has opposite sign at the $K$ and the $K^{\prime}$ points, which implies that the direction of the valley pseudospin polarization induced by the magnetic field cancel each other [14. In this case, the valley degeneracy is protected by spatial inversion symmetry [15]. Besides the usual spatial translation and rotation symmetries, pristine graphene Hamiltonian is invariant under a large number of symmetries in the isospin spaces [16]. By introducing the most general disorder Hamiltonian $H_{d i s}=\sum_{i, j} V_{i j} \sigma_{i} \tau_{j}$, pseudospin symmetries can be broken depending on which elements of $V_{i j}$ are non-zero ([17] and [18]). In particular, local diagonal disorder can be obtained experimentally by irradiation [19, where carbon atoms are extracted from the graphene plane and adatoms or adsorbed species attach to the graphene plane 20, or in which some carbon atoms are chemically substituted for other elements. These adsorbed particles can induce a local potential at the sites where they couple to the carbon lattice and change the on-site energy in the Hamiltonian. For weak random substitutional disorder potential, intravalley mixing within either the $K$ and $K^{\prime}$ valleys is possible, but not intervalley mixing. In the case of a random binary alloy disorder, a gap can be open in the Fermi level due to the broken $C_{z}$ chiral symmetry [16. The peculiar behavior of the $n=0$ Landau level in graphene, where its amplitude is nonzero only in one of the sublattices, namely, at $B$ sites for $K$ valley and $A$ sites for $K^{\prime}$ valley, combined with the random diagonal disorder can enhance the asymmetry in the valley amplitudes, which is related to the geometric nature of the Bloch band and its relation with Berry phase (see [21], 22], 223, [24] and [25]). This asymmetry can be detected by a population difference in the two valleys as a signal of orbital magnetization (see [11]) and in turn, this asymmetry can be enhanced by an asymmetry in the relative density of states for both valley pseudospins. In this sense, the aim of this work is to study the behavior of the valley pseudospin under a constant magnetic field and random binary disorder. In particular, self-energies and density of states for both sublattices and both valleys will be obtained by applying coherent potential approximation (CPA) (see [26, 27] and [28). In this method, the system is replaced by an effective medium with a complex self-energy that replace the disorder random potential. The value of the effective medium self-energy can be obtained by demanding that the average scattering of an electron by the surrounding medium is zero. Improvement of the method for better understanding of non local disorder can be done by replacing the single impurity site by a cluster of atoms (see 29], [30] and [31]). Due to the unbroken translational symmetry, the embedded cluster method has been applied (32], 33] and [34]). To obey all the imposed criteria for the CPA (see [35]), the Non Local Coherent Potential approximation has been developed [36] which is based in the approximation of continuous lattice functions $f(k)$ by cluster function $f\left(K_{n}\right)$, where $K_{n}$ are points in momentum space that satisfy Born-von Karman boundary conditions. Without taking into account the improvements of the CPA method, we will consider the results for low energy Bloch electrons with self-energies in the full Born approximation with nested diagrams included 1 This work will be organized as follow: In section II, the magnetic Green function with diagonal on-site energies will be computed for graphene. Although this procedure has been studied without diagonal on-site energies, for a self-contained work this results will be generalized. In section III, single-site approximation will be applied and a system

\footnotetext{
${ }^{1}$ See diagramatic techniques for CPA [37].
} 
of coupled Soven equation will be found and solved. The discussion of the results is shown in section IV and the principal findings of this paper are highlighted in the conclusion.

\section{Green function}

For a self-contained lecture of this paper, a brief introduction of the quantum mechanics of graphene in a constant magnetic field in the long wavelength approximation will be introduced (see [38]). The Hamiltonian in the two inequivalent corners of the Brillouin zones can be put in a compact notation as

$$
H_{0}^{(\lambda)}=v_{F}\left(\lambda \sigma_{x} p_{x}+\sigma_{y} p_{y}\right)+\left(\begin{array}{cc}
\Sigma_{A}^{(\lambda)} & 0 \\
0 & \Sigma_{B}^{(\lambda)}
\end{array}\right)
$$

where $\lambda=1$ is for the $K$ valley and $\lambda=-1$ for the $K^{\prime}$ valley. A diagonal energy matrix has been introduced for further application to single-site approximation (CPA). The quasiparticle momentum is $\mathbf{p}-e \mathbf{A}$, where $e$ is the electron charge and $\mathbf{A}$ is the vector potential which in the Landau gauge reads $\mathbf{A}=(-B y, 0,0)$ and $v_{F}=10^{6} \mathrm{~m} / \mathrm{s}$ is the Fermi velocity ${ }^{2}$ The Hamiltonian of last equation is invariant under traslations in the $x$ direction which means that the wave functions can be written as $\psi^{(\lambda)}=e^{i k x}\left(\psi_{A}^{(\lambda)}, \psi_{B}^{(\lambda)}\right)$. A coordinate transformation $\bar{y}=\hbar v_{F} k_{x}-e B y$ can be applied followed by a scale transformation $\bar{y}=\frac{1}{\sqrt{e \hbar B}} \overline{\bar{y}}$, finally, introducing the annihilation and creation operators $a=\frac{1}{\sqrt{2}}\left(\overline{\bar{y}}+\frac{\partial}{\partial \overline{\bar{y}}}\right)$ and $a^{\dagger}=\frac{1}{\sqrt{2}}\left(\overline{\bar{y}}-\frac{\partial}{\partial \overline{\bar{y}}}\right)$, the Hamiltonian of eq.(2) reads

$$
H^{(\lambda=1)}=\left(\begin{array}{cc}
\Sigma_{A}^{(1)} & \gamma a \\
\gamma a^{\dagger} & \Sigma_{B}^{(1)}
\end{array}\right) \quad H^{(\lambda=-1)}=\left(\begin{array}{cc}
\Sigma_{A}^{(-1)} & -\gamma a^{\dagger} \\
-\gamma a & \Sigma_{B}^{(-1)}
\end{array}\right)
$$

where $\gamma=v_{F} \sqrt{2 e \hbar B}$. The eigenfunctions and eigenvectors for the Hamiltonian of last equation reads (see [39] and [40])

$$
\psi_{(n, s, k)}^{(\lambda)}(r)=e^{i k x} \frac{C_{n}}{\sqrt{2 L}} \varphi_{(n, s, k)}^{(\lambda)}(\xi)
$$

where $\varphi_{(n, s, k)}^{(\lambda)}(\xi)$ reads

$$
\varphi_{(n, s, k)}^{(1)}(\xi)=\left(\begin{array}{c}
\alpha_{n}^{(1, s)} \phi_{n-1, k}(\xi)\left(1-\delta_{n, 0}\right) \\
\phi_{n, k}(\xi)
\end{array}\right) \quad \varphi_{(n, s, k)}^{(-1)}(\xi)=\left(\begin{array}{c}
\phi_{n, k}(\xi) \\
\alpha_{n}^{(-1, s)} \phi_{n-1, k}(\xi)\left(1-\delta_{n, 0}\right)
\end{array}\right)
$$

being $\phi_{n, k}(\xi)$ the wave function of the harmonic oscillator ${ }^{3}$

$$
\phi_{n, k}(\xi)=\frac{\pi^{-1 / 4}}{\sqrt{2^{n} n !}} e^{-\frac{1}{2} \xi^{2}} H_{n, k}(\xi)
$$

$\xi=\frac{\overline{\bar{y}}}{l_{B}}-l_{B} k, L=\sqrt{A}$ where $A$ is the area of the graphene sheet and

$$
\alpha_{n}^{(\lambda, s)}=\frac{\lambda\left(\Sigma_{A}^{(\lambda)}-\Sigma_{B}^{(\lambda)}\right)-s \sqrt{\left(\Sigma_{A}^{(\lambda)}-\Sigma_{B}^{(\lambda)}\right)^{2}+4 \gamma^{2} n}}{2 \gamma \sqrt{n}}
$$

\footnotetext{
${ }^{2}$ Spin Zeeman energy are completely neglected because the spin splitting is much smaller than Landau-level separations.

${ }^{3}$ The factor $\left(1-\delta_{n, 0}\right)$ is introduced to discriminate the wave function with $n=0$. In this case, only one sublattice contributes in both valleys $K$ and $K^{\prime}$.
} 
where $s= \pm 1$ is the conduction (valence) band index. The coefficient $C_{n}$ is $C_{n}=\frac{1}{\sqrt{2-\delta_{n, 0}}}$ and $l_{B}=\sqrt{\hbar / e B}$ is the magnetic length. The eigenvalues of the Hamiltonian reads

$$
E_{n}^{(s)}=\frac{\Sigma_{A}^{(\lambda)}+\Sigma_{B}^{(\lambda)}-s \sqrt{\left(\Sigma_{A}^{(\lambda)}-\Sigma_{B}^{(\lambda)}\right)^{2}+4 \gamma^{2} n}}{2}
$$

The degeneracy of each level is $g=L^{2} B / \phi_{0}$ where $\phi_{0}=\hbar / e$ is the quantum of flux. The low energy description is only valid as long as the characteristic energy of the excitations is not larger than an energy cutoff $E_{n, 1}<E_{C}$, where $E_{C}=\hbar v_{F} k_{\Delta}$ and $k_{\Delta}$ is a momentum cutoff. We can choose $k_{\Delta}$ in such a way to conserve the total number of states in the Brillouin zone, that is, $\pi k_{\Delta}^{2}=(2 \pi)^{2} / A_{C}$, where $A_{C}=3 \sqrt{3} a^{2} / 2$ is thea area of the hexagonal lattice (see [41]). Then, using eq.(77), $E<E_{C}$ implies that $n<\frac{1}{2 e \hbar B}\left[\frac{\delta^{2}}{a}-\frac{\delta\left(\Sigma_{A}^{(\lambda)}+\Sigma_{B}^{(\lambda)}\right)}{a v_{F}}+\frac{\Sigma_{A} \Sigma_{B}}{v_{F}^{2}}\right]$ where $\delta=\sqrt{8 \pi / 3 \sqrt{3}}$, then for weak magnetic fields, the cutoff tends to infinity and for high magnetic fields, the cutoff tends to zero. Eq. (7) indicates that asymmetry in the substitutional impurity energies opens an energy gap $\Delta_{n}^{(\lambda)}=\sqrt{\left(\Sigma_{A}^{(\lambda)}-\Sigma_{B}^{(\lambda)}\right)^{2}+4 \gamma^{2} n}$.

Using the spectral representation, the Green function of this system reads

$$
\begin{gathered}
g_{i j}^{(\lambda)}\left(r, r^{\prime}, E\right)=\frac{1}{2 L} \sum_{n=0}^{+n_{\Delta}} \sum_{s= \pm 1} \int_{-\infty}^{+\infty} \\
\frac{\left(\alpha_{n}^{(\lambda, s)}\right)^{|\lambda+1-i-j|} e^{i k\left(x-x^{\prime}\right)} \phi_{n-i+\frac{1-\lambda}{2}, k}^{*}\left(\xi^{\prime}\right) \phi_{n-j+\frac{1-\lambda}{2}, k}(\xi)\left(1-\delta_{n, 0}\right)^{|\lambda+1-i-j|} d k}{\left(2-\delta_{n, 0}\right)\left(E-E_{n}^{(s)}\right)}
\end{gathered}
$$

where for the moment $i, j=0,1$ where $0(1)$ represent the sublattice $A(B)$. We can perform the integration in $k$ by using eq.(5) and completing squares

$$
e^{i k\left(x-x^{\prime}\right)} e^{-\frac{1}{2}\left(\frac{\overline{\bar{y}}}{l_{B}}-l_{B} k\right)^{2}} e^{-\frac{1}{2}\left(\frac{\overline{\bar{y}}^{\prime}}{l_{B}}-l_{B} k\right)^{2}}=e^{-l_{B}^{2}\left(k-\frac{\overline{\bar{y}}+\bar{y}^{\prime}+i\left(x-x^{\prime}\right)}{2 l_{B}^{2}}\right)^{2}} e^{-\frac{1}{4 l_{B}^{2}\left[\left(\overline{\bar{y}}-\bar{y}^{\prime}\right)^{2}+\left(x-x^{\prime}\right)^{2}\right]+\frac{i}{2 l_{B}^{2}}\left(\overline{\bar{y}}+\bar{y}^{\prime}\right)\left(x-x^{\prime}\right)}}
$$

Introducing the following coordinate transformation

$$
q=-l_{B} k+\frac{\overline{\bar{y}}+\overline{\bar{y}}^{\prime}+i\left(x-x^{\prime}\right)}{2 l_{B}}
$$

and using the following relation (see eq. $\left(7.377^{8}\right)$ of page 804 of [42]) the Green function reads

$$
\begin{gathered}
g_{i j}^{(\lambda)}\left(r, r^{\prime}, E\right)=\frac{(-1)^{j-i} f\left(r, r^{\prime}\right)^{j-i} e^{-\frac{\rho^{2}}{2}+i \eta\left(r, r^{\prime}\right)}}{2 L} \times \\
\sum_{n=0}^{+n_{\Delta}} \frac{\left(1-\delta_{n, 0}\right)^{|\lambda+1-i-j|}}{\left(2-\delta_{n, 0}\right)} T_{n}^{(\lambda, i, j)} \sqrt{\frac{2^{j-i}\left(n-j+\frac{1-\lambda}{2}\right) !}{\left(n-i+\frac{1-\lambda}{2}\right) !}} L_{n-j+\frac{1-\lambda}{2}}^{|j-i|}\left(\rho^{2}\right)
\end{gathered}
$$

where the sum in $s$ has been performed and where

$$
f\left(r, r^{\prime}\right)=\frac{\overline{\bar{y}}-\overline{\bar{y}}^{\prime}-i\left(x-x^{\prime}\right)}{2 l_{B}}
$$

$\eta\left(r, r^{\prime}\right)$ is a gauge term that reads

$$
\eta\left(r, r^{\prime}\right)=\frac{1}{2 l_{B}^{2}}\left(\overline{\bar{y}}+\overline{\bar{y}}^{\prime}\right)\left(x-x^{\prime}\right)
$$

and

$$
\rho^{2}=\frac{\left|r-r^{\prime}\right|^{2}}{2 l_{B}^{2}}
$$


The $T_{n}^{(\lambda, i, j)}$ matrix elements read 4

$$
\begin{gathered}
T_{n}^{(|\lambda|, 0,0)}=T_{n}^{(-|\lambda|, 1,1)}=\frac{-2 E n \gamma^{2}+\left(2 n \gamma^{2}+E Q_{1}^{(\lambda)}-Q_{2}^{(\lambda)}\right) Q_{1}^{(\lambda)}-Q_{2}^{(\lambda)} E+Q_{3}^{(\lambda)}}{n \gamma^{2}\left[-E^{2}+n \gamma^{2}+\Sigma_{A}^{(\lambda)}\left(E-\Sigma_{B}^{(\lambda)}\right)+E \Sigma_{B}^{(\lambda)}\right]} \\
T_{n}^{(\lambda, 1,0)}=T_{n}^{(\lambda, 0,1)}=\frac{-2 n \gamma^{2}-\lambda\left(\Sigma_{A}^{(\lambda)}-\Sigma_{B}^{(\lambda)}\right)\left[E+Q_{1}^{(\lambda)}-\Sigma_{B}^{(\lambda)}-\Sigma_{A}^{(\lambda)}\right]}{\gamma \sqrt{n}\left[n \gamma^{2}-E^{2}+\Sigma_{A}^{(\lambda)}\left(E-\Sigma_{B}^{(\lambda)}\right)+E \Sigma_{B}^{(\lambda)}\right]} \\
T_{n}^{(1,1,1)}=T_{n}^{(-1,0,0)}=\frac{-2 E+\Sigma_{A}^{(\lambda)}+\Sigma_{B}^{(\lambda)}}{n \gamma^{2}-E^{2}+\Sigma_{A}^{(\lambda)}\left(E-\Sigma_{B}^{(\lambda)}\right)+E \Sigma_{B}^{(\lambda)}}
\end{gathered}
$$

where

$$
Q_{i}^{(\lambda)}=\frac{1}{2}\left[(1+\lambda)\left(\Sigma_{A}^{(\lambda)}\right)^{i}-(\lambda-1)\left(\Sigma_{B}^{(\lambda)}\right)^{i}\right]
$$

in the case that $\Sigma_{A}^{(\lambda)}=\Sigma_{B}^{(\lambda)}=0$, eq.(11) is identical to eq.(11) and eq.(12) for $\lambda=1$ of [43. It should be pointed out that, as it was shown in eq.(44) the application of a magnetic field to graphene allows to obtain different wavefunctions for sublattice $A$ and $B$ in each valley. This is different of what occurs when $B$ is zero in the effective low-energy description, because in this case the wavefunctions in each sublattice site differ only by a phase which depends on the polar angle of the wave vector $\mathbf{k}$. For this, the probability amplitudes in both sublattice sites are identical. This crucial difference is of major importance when diagonal disorder is introduced because it will increase the availability of states near the Fermi energy without disabling the sublattice asymmetry introduced by the magnetic field.

\section{Single-site approximation}

To apply CPA we can introduce impurity potentials in the $A$ and $B$ sublattices in the following form

$$
H=v_{F}\left(\lambda \sigma_{x} p_{x}+\sigma_{y} p_{y}\right)+\left(\begin{array}{cc}
V_{A} & 0 \\
0 & V_{B}
\end{array}\right)
$$

where the potential $V^{A / B}$ reads

$$
V^{A / B}=\sum_{r_{i}}^{N_{A / B}} V_{i}^{A / B}\left|r_{i}\right\rangle\left\langle r_{i}\right|
$$

and $V_{i}^{A / B}$ are the on-site impurity energies with a probability distribution $P\left(V_{i}^{A / B}\right)$ which is the same for both sublattices and pseudospin valleys 5 To apply CPA, we can introduce an effective Hamiltonian

$$
H_{e f}=v_{F}\left(\lambda \sigma_{x} p_{x}+\sigma_{y} p_{y}\right)+\Sigma^{(\lambda)}(z)
$$

where $\Sigma(z)$ is a energy-dependent self-energy matrix which reads

$$
\Sigma^{(\lambda)}(z)=\left(\begin{array}{cc}
\Sigma_{A}^{(\lambda)}(z) & 0 \\
0 & \Sigma_{B}^{(\lambda)}(z)
\end{array}\right)
$$

Then, an impurity can be introduced in the effective medium in an specific site $r_{0}$ by defining the following Hamiltonian

$$
H_{h}=H_{e f}+W\left|r_{0}\right\rangle\left\langle r_{0}\right|
$$

where $W$ reads

$$
W=\left(\begin{array}{cc}
V_{A}-\Sigma_{A}(z) & 0 \\
0 & V_{B}-\Sigma_{B}(z)
\end{array}\right)
$$

\footnotetext{
${ }^{4}$ The $T_{n}^{(\lambda, i, j)}$ coefficients have been introduced to write eq. (11) in a more compact way, although its units are $E^{-1}$ which are the units of the Green function.

${ }^{5}$ The random distribution is identical for $A$ and $B$ sublattices.
} 
Applying the self-consistency condition for the Green function $G_{e f}=\left\langle G_{h}\right\rangle$, where $\langle\ldots\rangle$ means configurational averaging, we obtain the Soven equation in matrix form 6

$$
\left\langle W\left[I-g_{e f}\left(r_{0}, r_{0}\right) W\right]^{-1}\right\rangle=0
$$

where $g_{e f}$ is the Green function matrix with magnetic field associated to the effective Hamiltonian of eq.(19), which is identical to eq.(11) and implies that the effective Green function is that of eq.(11), which must be evaluated at $r_{0}$ in both coordinates arguments

$$
g_{i j}^{(\lambda)}\left(r_{0}, r_{0}, E\right)=-\delta_{i j} \frac{1}{2 L} \sum_{n=0}^{+n_{\Delta}} \frac{\left(1-\delta_{n, 0}\right)^{|\lambda+1-i-j|}}{\left(2-\delta_{n, 0}\right)} T_{n}^{(\lambda, i, j)}
$$

The non-diagonal elements are zero due to the factor $f(r, r)=0$ for identical points. This result implies that eq.(23) contains a system of two coupled self-energies that must be solved self-consistently. Applying the configurational averaging by using the probability distribution for random binary alloy $P\left(V_{i}\right)=c \delta\left(V-\epsilon_{1}\right)+(1-c) \delta\left(V-\epsilon_{2}\right)$, the system of eq. (23) reads

$$
\begin{aligned}
& \frac{c\left(\epsilon_{1}-\Sigma_{A}^{(\lambda)}\right)}{1-g_{A A}^{(\lambda)}\left[\epsilon_{1}-\Sigma_{A}^{(\lambda)}\right]}+\frac{(1-c)\left(\epsilon_{2}-\Sigma_{A}^{(\lambda)}\right)}{1-g_{A A}^{(\lambda)}\left[\epsilon_{2}-\Sigma_{A}^{(\lambda)}\right]}=0 \\
& \frac{c\left(\epsilon_{1}-\Sigma_{B}^{(\lambda)}\right)}{1-g_{B B}^{(\lambda)}\left[\epsilon_{1}-\Sigma_{B}^{(\lambda)}\right]}+\frac{(1-c)\left(\epsilon_{2}-\Sigma_{B}^{(\lambda)}\right)}{1-g_{B B}^{(\lambda)}\left[\epsilon_{2}-\Sigma_{B}^{(\lambda)}\right]}=0
\end{aligned}
$$

where we have restored the original notation $g_{A A}^{(\lambda)}=g_{00}^{(\lambda)}$ and $g_{B B}^{(\lambda)}=g_{11}^{(\lambda)}$, where these functions depend on $\Sigma_{A}^{(\lambda)}$ and $\Sigma_{B}^{(\lambda)}$ and $c$ is the concentration of $\epsilon_{1}$ impurities that can be located in $A$ or $B$ sublattices and $\epsilon_{2}$ is the on-site impurity energy with probability $1-c$ that can be located in the $A$ or $B$ sublattice.

\section{Results and discussion}

To apply CPA to eq. 225) we have to take into account the diagonal elements of the Green function, which can be solved exactly

$$
\begin{gathered}
g_{A A}^{(1)}(r, r, E)=g_{B B}^{(-1)}\left(r_{0}, r_{0}, E\right)= \\
-\frac{1}{4 L} A^{(\lambda)}\left[\psi^{(0)}\left(n_{\Delta}+1+C^{(\lambda)}\right)-\psi^{(0)}\left(1+C^{(\lambda)}\right)\right] \\
-\frac{1}{4 L} \frac{B^{(\lambda)}}{C^{(\lambda)}}\left[\psi^{(0)}\left(1+C^{(\lambda)}\right)+\psi^{(0)}\left(n_{\Delta}+1\right)-\psi^{(0)}\left(C^{(\lambda)}+n_{\Delta}+1\right)+\gamma_{0}\right]
\end{gathered}
$$

where $\gamma_{0}$ is the Euler-Mascheroni constant, $\psi^{(0)}$ is the Digamma function and the coefficients $A, B$ and $C$ reads

$$
\begin{gathered}
A^{(\lambda)}(E)=2 \gamma^{-2}\left[Q_{1}^{(\lambda)}-E\right] \\
B^{(\lambda)}(E)=\gamma^{-4}\left[\left(E Q_{1}^{(\lambda)}-Q_{2}^{(\lambda)}\right) Q_{1}^{(\lambda)}-Q_{2}^{(\lambda)} E+Q_{3}^{(\lambda)}\right] \\
C^{(\lambda)}(E)=\gamma^{-2}\left[-E^{2}+\Sigma_{A}^{(\lambda)}\left(E-\Sigma_{B}^{(\lambda)}\right)+E \Sigma_{B}^{(\lambda)}\right]
\end{gathered}
$$

\footnotetext{
${ }^{6}$ For more details about CPA see [35, chapter VIII.
} 


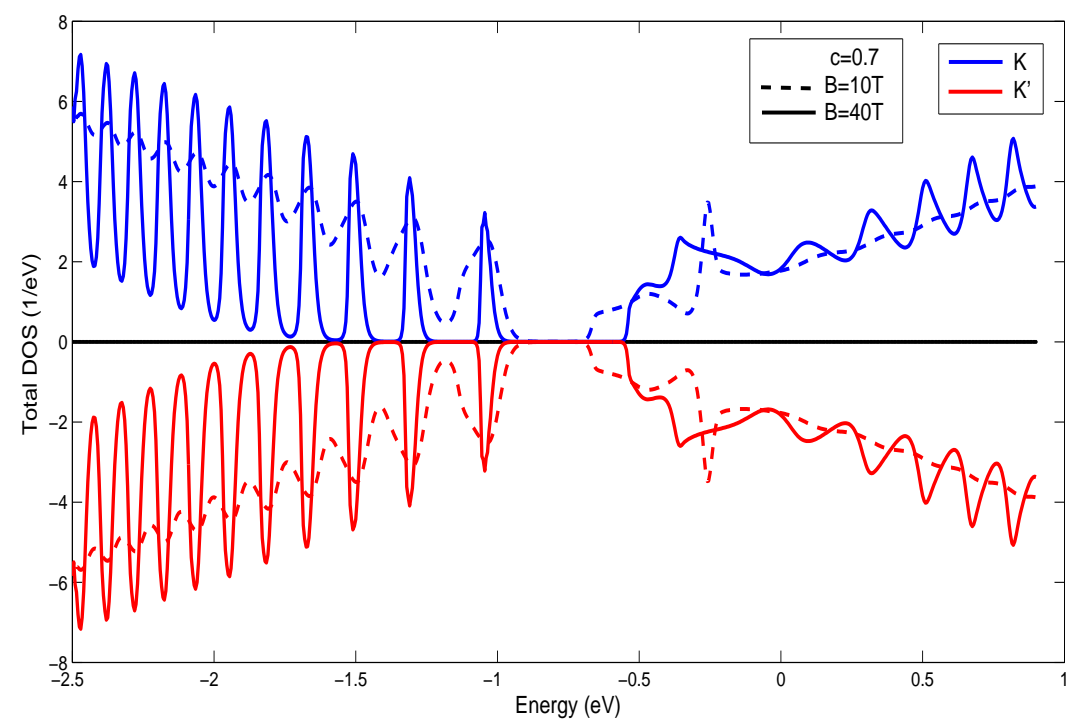

Figure 1: Total density of states for two different values of applied magnetic field. Blue line for $K$ valley and red line for $K^{\prime}$ valley and $\epsilon_{1}=-0.5=-\epsilon_{2}$.

In the same way

$$
\begin{gathered}
g_{B B}^{(1)}(r, r, E)=g_{A A}^{(-1)}(r, r, E)= \\
-\frac{1}{2 L} \frac{-2 E+\Sigma_{A}^{(\lambda)}+\Sigma_{B}^{(\lambda)}}{-E^{2}+\Sigma_{A}^{(\lambda)}\left(E-\Sigma_{B}^{(\lambda)}\right)+E \Sigma_{B}^{(\lambda)}} \\
-\frac{1}{4 L} D^{(\lambda)}\left[\psi^{(0)}\left(F^{(\lambda)}+n_{\Delta}+1\right)-\psi^{(0)}\left(1+F^{(\lambda)}\right)\right]
\end{gathered}
$$

where

$$
\begin{gathered}
D^{(\lambda)}(E)=\gamma^{-2}\left[-2 E+\Sigma_{A}^{(\lambda)}+\Sigma_{B}^{(\lambda)}\right] \\
F^{(\lambda)}(E)=\gamma^{-2}\left[-E^{2}+\Sigma_{A}^{(\lambda)}\left(E-\Sigma_{B}^{(\lambda)}\right)+E \Sigma_{B}^{(\lambda)}\right]
\end{gathered}
$$

From eq.(26) and eq.(28), the contribution of the conduction (valence) band in the sublattice $A(B)$ equal the valence (conduction) band in the sublattice $B(A)$, which reflects the fact that, although on-site impurities were introduced, the configurational averaging restore the full symmetry of the Hamiltonian. A convenient way to treat the coupled system of Soven equations of eq.(25) is to consider an iterative formula equivalent to the CPA condition in the following way

$\Sigma_{A / B}^{(\lambda)}(i+1)=\Sigma_{A / B}^{(\lambda)}(i)+\frac{1}{g_{A A / B B}^{(\lambda)}\left(\Sigma_{A / B}^{(\lambda)}(i), \Sigma_{B / A}^{(\lambda)}(i)\right)}+\left\langle\frac{1}{\Sigma_{A / B}^{(\lambda)}(i)-V+\frac{1}{g_{A A / B B}^{(\lambda)}\left(\left(\Sigma_{A / B}^{(\lambda)}(i), \Sigma_{B / A}^{(\lambda)}(i)\right)\right)}}\right\rangle^{-1}$

In order to obtain the value of the self-energies, the iteration described above for both sublattices is repeated until $\left|\Sigma_{A / B}^{(\lambda)}(i+1)-\Sigma_{A / B}^{(\lambda)}(i)\right|<\varepsilon$, where $\varepsilon$ determines the requested precision of the calculation. The total and partial density of states can be computed as

$$
\rho(E)=-\frac{1}{\pi} \operatorname{Im} g(E-\Sigma(E))
$$



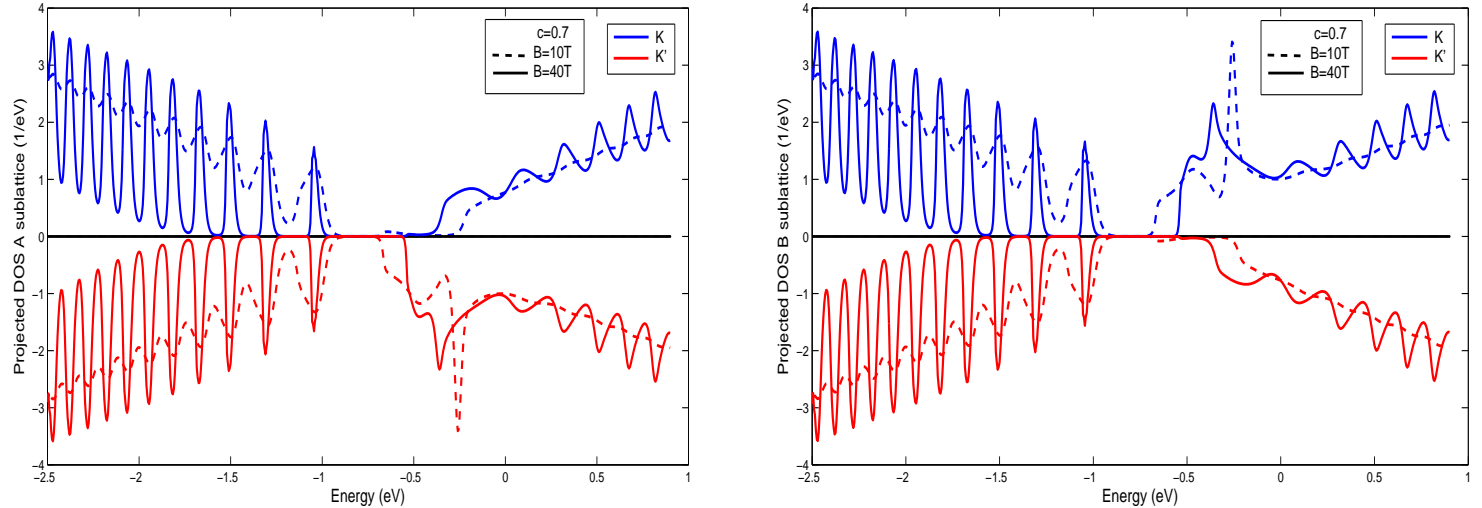

Figure 2: Projected DOS on A sublattice for two different values of magnetic field. Blue line for $K$ valley and red line for $K^{\prime}$ valley and $\epsilon_{A}=-0.5=$ $-\epsilon_{B}$
Figure 3: Projected DOS on B sublattice for two different values of magnetic field. Blue line for $K$ valley and red line for $K^{\prime}$ valley and $\epsilon_{A}=-0.5=$ $-\epsilon_{B}$

In figure 1 and figures 2 and 3 the total density of states and projected DOS in $A$ and $B$ sublattice is shown for both pseudospin valleys using $\epsilon_{1}=-0.5=-\epsilon_{2}, c=0.7$ and for $B=10 T$ and $B=40 T$. For the specific concentration chosen, we are considering acceptor impurities. The total DOS shows effectively that no broken pseudospin symmetry has been produced by averaging over disorder, but figure 2 and 3 shows an asymmetry in the $K$ and $K^{\prime}$ valleys for the projected density of states below the Fermi level in both sublattices. There is an enhancement of the population for $A$ sublattice in the $K$ valley with respect to the $K^{\prime}$ valley and the opposite for $B$ sublattice. This behavior is expected due to the asymmetry of the lowest Landau level in both sublattices, where the shifted peak correspond to the impurity contribution. The behavior of the density of states near the energy threshold where the approximation holds is expected and reflects the linear regime with the peaks corresponding to the broadening of Landau levels (see figure 10 of [41]). In turn, the impurities open an energy gap at the Dirac point (see 44) which lies below the Fermi level for both magnetic field strength, although for higher $B$, the impurity band is reduced and shifted away the Fermi level. In the projected DOS figures, the energy gap introduced by the diagonal impurities is different for $K$ and $K^{\prime}$ valley, which is a consequence of the self-energy asymmetry results obtained from CPA. In the case that $c=0.5$, the asymmetry in the gap for $K$ and $K^{\prime}$ valleys should be of major importance because in this case, the impurity band will be located in the Fermi level and for half-filling, a net magnetization will appears 7 In figure 4 and 5, the net magnetization in both sublattices is shown for different impurity on-site energies where

$$
\mu_{A / B} / \mu_{0}=\int_{-E_{C}}^{E_{F}}\left[\rho_{A / B}^{K}(E)-\rho_{A / B}^{K^{\prime}}(E)\right] d E
$$

where $\mu_{0}$ is the Bohr magneton. As it is expected, both magnetization are equal an opposite, which implies that there is no net magnetization when both sublattices are taking into account. In both sublattices, the magnetization present a maximum value that decreases with an increasing on-site impurity potentials, which implies that it is always possible to maximize magnetization with an specific choice of the parameters. The shift of the maximum with the displacement of impurity on-site energy is the combination of two physical process: the enhanced asymmetry between $K$ and $K^{\prime}$ density of states near the Fermi level when the strength of the impurities is raised and the spreadening of the impurity band due to the weak magnetic field, although when electron many body effects are taking

\footnotetext{
${ }^{7}$ The $c=0.5$ impurity concentration has not been studied in this work because convergence for self-energy in CPA is difficult to achieve in this case.
} 

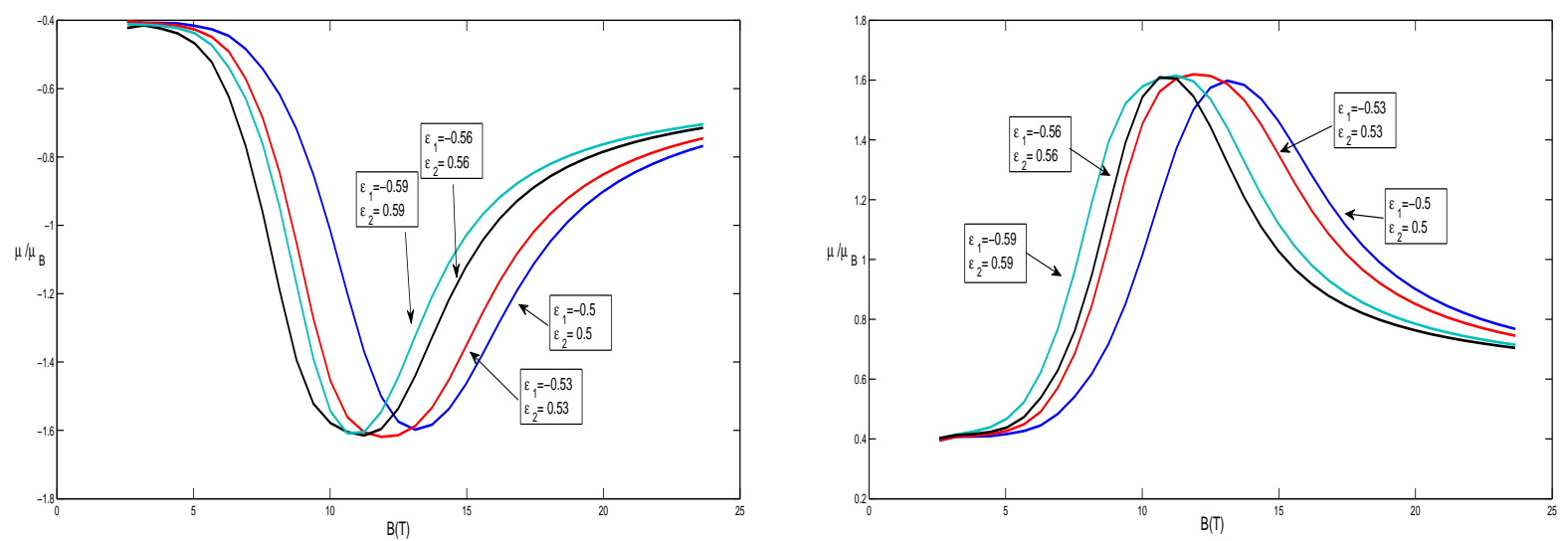

Figure 4: Magnetization of A sublattice due to relative Figure 5: Magnetization of B sublattice due to relative difference between density of state contributions for difference between density of state contributions for the two valleys pseudospin. the two valleys pseudospin.

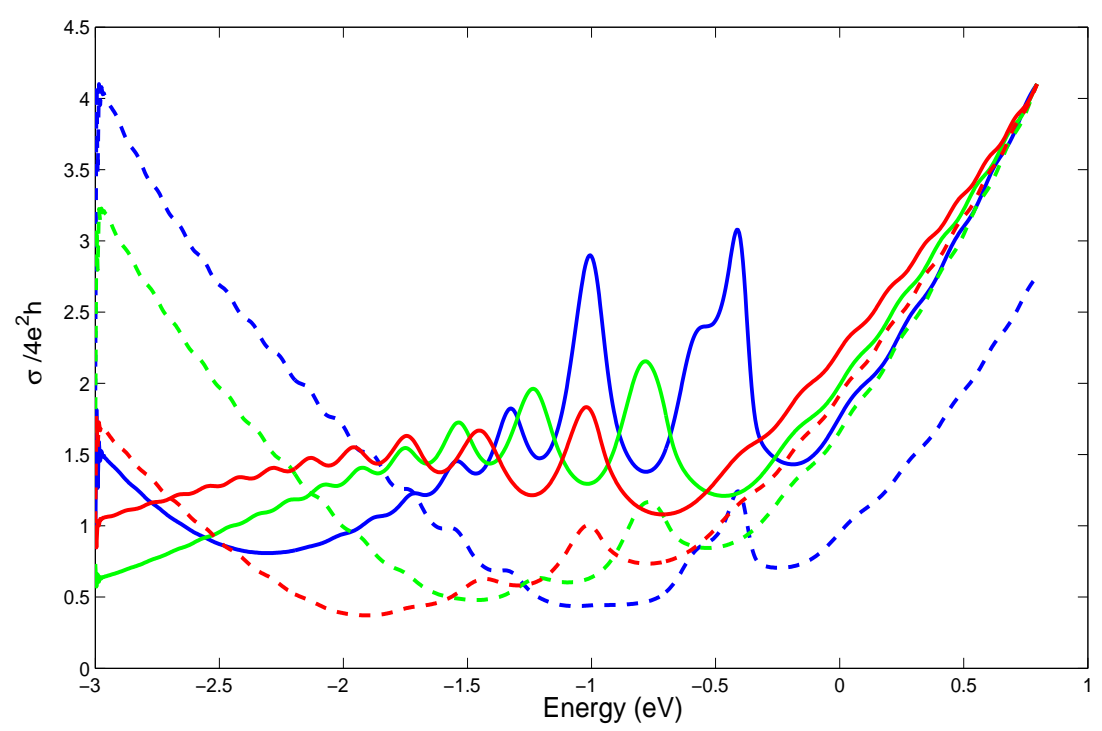

Figure 6: Conductivity as a function of energy for different on-site impurities. Blue line $\epsilon_{1}=\epsilon_{2}=-0.6$, green line $\epsilon_{1}=\epsilon_{2}=-0.8$ and red line for $\epsilon_{1}=\epsilon_{2}=-1$. Dashed line for sublattice $A$ scattering contribution to conductivity. The magnetic field strength used is $B=10 T$. 


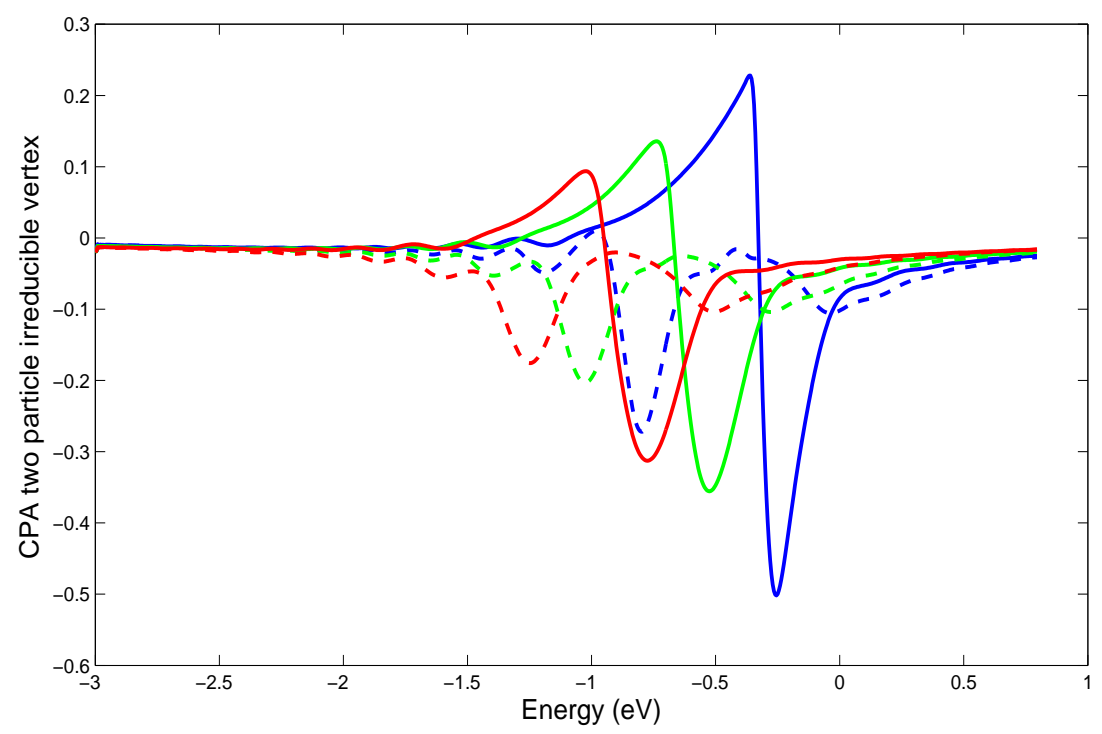

Figure 7: Vertex function as a function of energy for different on-site impurities. Blue line $\epsilon_{1}=\epsilon_{2}=$ -0.6 , green line $\epsilon_{1}=\epsilon_{2}=-0.8$ and red line for $\epsilon_{1}=\epsilon_{2}=-1$. Thick line for sublattice $A$ scattering and dashed line for sublattice $B$ scattering contribution to conductivity. The magnetic field strength used is $B=10 T$.

into account, this behavior should be reduced because high density of states available from the $K$ or $K^{\prime}$ band increase the energy caused by spin-alignment which in turn results in a small contribution to the exchange energy.

In figure 6. the conductivity is plotted against energy using eq.(14) of 45, where Kubo formalism is used, for different values of impurity on-site energies. The position of the conductivity peaks correspond to the minimum and maximum of the energy band, where the density of states is the largest. In dashed line, the contribution to conductivity for sublattice $A$ scattering is taking into account. In particular, for higher values of impurity on-site energies, the contribution to total conductivity peaks are given by $A$ sublattice scattering (red line), but is not the case for the blue line and green line, where there is a contribution from $B$ sublattice scattering for the second peak respectively. In figure 7 the local two-particle irreducible vertex $\lambda$ consistent with the coherent potential approximation for the self-energy $\Sigma$ is plotted against energy, for retarded and advanced Green functions (see [46], 47], [48] and [49]) with the same parameters as figure 6 The peak of the vertex for $A$ sublattice scattering (blue thick line in figure 7) is related to peak near the Fermi level in figure 6, while the second peak is given by the contribution of vertex for $B$ sublattice scattering (blue dashed line in figure 7) with its respective decrease in the conductivity contribution (see blue dashed line in figure 6). In turn, from figure 7. the vertex function for $A$ sublattice scattering shows a singular behavior where the retarded and advanced Green function in the real part of the energy are identical. The same behavior is not present for the $B$ sublattice scattering which is related to the different contributions of Landau levels for $A$ and $B$ sublattice. The singular behavior of the vertex function implies a critical contribution to conductivity that change sign and can be related to weak anti-localization (see [50, [51, [52], [53. and [54]).

These results reflect the fact that, although a simple model has been considered, where the impurities are diagonal in the sublattice basis and short-ranged and averaging has been applied, the application of a constant magnetic field introduce subtleties in the valley properties of electrons. In particular, the different Landau levels contributions to the eigenfunctions for each sublattice increase 
the asymmetry in the orbital magnetic moment present in electrons in the two Dirac points and for particular values of impurity concentrations and magnetic field strength, the net magnetization could be larger to create and manipulate polarized valley currents. From the experimental viewpoint, doping in graphene can be obtained through electric doping by changing gate voltage (see [55] and [56]) or by chemical doping, which is discussed as surface transfer doping and subtitutional doping (see [57] and [58]). For a general review of the experimental procedure to obtain doping asymmetry in graphene see [59, 60] and the references therein and for the direct experimental determination of the chemical bonding of individual impurity atoms see [61]. In the case that p-type and n-type doping can be achieved in a controled way and where the experimental determination of the on-site energies results in slightly different values, then it would be possible to tune up population difference in both valleys in each sublattice site through the magnetic field strength and valley currents could be obtained by applying a gate voltage.

\section{Conclusion}

We have investigated the behavior of the density of states in both sublattices and for the different valley pseudospins in graphene with disorder and magnetic field in the low energy effective-mass theory. By applying coherent potential approximation for a binary random alloy and considering the contribution for both sublattices on the effective self-energies, a coupled system of Soven equations can be obtained to be solved numerically. We have shown that a band gap is opened by an asymmetric on-site impurity concentration and the graphene electrons acquire an anomalous magnetic moment, which is opposite in different valleys, similar to a real spin. The valley contributions to the projected density of states for each sublattice are not simetrical and the differences depend highly in the interplay between the impurity band, the band edges and the broadening of the Landau levels. In the case that spinorbit interaction and Zeeman effect are considered, the asymmetry between valleys can be enhanced by splitting of Landau levels. In turn, conductivity and CPA local two-irreducible vertex has been computed showing that scattering from sublattice $A$ and $B$ do not contribute equally, which implies that graphene Bloch electrons correlations in the long wavelength approximation in averaged disorder and magnetic field develop unusual behavior for critical energy values.

\section{Acknowledgment}

This paper was partially supported by grants of CONICET (Argentina National Research Council) and Universidad Nacional del Sur (UNS) and by ANPCyT through PICT 1770, and PIP-CONICET Nos. 114-200901-00272 and 114-200901-00068 research grants, as well as by SGCyT-UNS., E.A.G., P.V.J. and J. S. A. are members of CONICET. P.B. is a fellow researcher at this institution. We are grateful to the Abdus Salam International Centre for Theoretical Physics (ICTP) to allow J. S. A. to spend his time in the Condensed Matter Section and to be benefited from helpul discussions with the staff.

\section{Author contributions}

All authors contributed equally to all aspects of this work.

\section{References}

[1] K. S. Novoselov, A. K. Geim, S. V. Morozov, D. Jiang, M. I. Katsnelson, I. V. Grigorieva, S. V. Dubonos and A. A. Firsov, Nature, 438, 197 (2005).

[2] A.K. Geim and K. S. Novoselov, Nature Materials, 6, 183 (2007). 
[3] Y. B. Zhang, Y.W. Tan, H. L. Stormer and P. Kim, Nature, 438, 201 (2005).

[4] A. H. Castro Neto, F. Guinea, N. M. R. Peres, K. S. Novoselov and A. K. Geim, Rev. Mod. Phys., 81, 109 (2009).

[5] M. O. Goerbig, Rev. Mod. Phys., 83, 4, (2011).

[6] J. McClure, Phys. Rev., 104, 666, (1956).

[7] S. Kuru, J. Negro and L. M. Nieto, J. Phys.: Condens. Matter, 21, 455305 (2009).

[8] Y. Zheng and T. Ando, Phys. Rev. B, 65, 245420 (2002).

[9] A. Rycerz, J. TworzydÃlo, and C. W. J. Beenakker, Nat. Phys., 3, 172-175, (2007).

[10] P. Recher, B. Trauzettel, A. Rycerz, Y. M. Blanter, C. W. J. Beenakker and A. F. Morpurgo, Phys. Rev. B, 76, 235404, (2007).

[11] D. Xiao, W. Yao and Q. Niu, Phys. Rev. Lett., 99, 236809, (2007).

[12] D. V. Bulaev, B. Trauzettel and D. Loss, Phys. Rev. B, 77, 235301, (2008).

[13] L. L. Foldy and S. A. Wouthuysen, Phys. Rev., 78, 29, (1950).

[14] K. Sasaki and R. Sato, Prog. Theor. Phys. Supplement, 176, 253-278, (2008).

[15] M. Taillerfumier, V. K. Dugaev, B. Canals, C. Lacroix and P. Bruno, Phys. Rev. B, 84, 085427, (2011).

[16] P. M. Ostrovsky, I. V. Gornyi and A. D. Mirlin, Eur. Phys. J. Special Topic, 148, 63-72 (2007).

[17] S. V. Morozov, K. S. Novoselov, M. I. Katsnelson, F. Schedin, L. A. Ponomarenko, D. Jiang, and A. K. Geim, Phys. Rev. Lett., 97, 016801 (2006).

[18] E. McCann and V. I. Fal'ko, Phys. Rev. B., 71, 085415 (2005).

[19] P. Esquinazi, D. Spemann, R. Hhne, A. Setzer, K.-H. Han and T. Butz, Phys. Rev. Lett., 91, 227201 (2003).

[20] F. Schedin, A. K. Geim, S. V. Morozov, D. Jiang, E. H. Hill, P. Blake, and K. S. Novoselov, Nature Materials, 6, 652 (2007).

[21] M. C. Chang and Q. Niu, Phys. Rev. B, 53, 7010, (1996).

[22] D. Xiao, J. Shi and Q. Niu, Phys. Rev. Lett., 95, 137204, (2005).

[23] T. Thonhauser, D. Ceresoli, D. Vanderbilt and R. Resta, Phys. Rev. Lett., 95, 137205, (2005).

[24] D. Ceresoli, T. Thonhauser, D. Vanderbilt and R. Resta, Phys. Rev. B, 74, 024408, (2006).

[25] D. Xiao, M. C. Chang and Q. Niu, Rev. Mod. Phys., 82, 1959, (2010).

[26] P. Soven, Phys. Rev., 156, 839 (1967).

[27] P. Soven, Phys. Rev., 178, 1136 (1969).

[28] B. Velicky, S. Kirkpatrick and H. Ehrenreich, Phys. Rev., 175, 747 (1968).

[29] M. Tsukada. J. Phys. Soc. Jpn, 32:1475, (1972).

[30] A. R. Bishop and A. Mookerjee. J. Phys. C., 7:2165 (1973). 
[31] F. Ducastelle. J. Phys. C, 8:3297 (1975).

[32] A. Gonis and J. W. Garland, Phys. Rev. B, 16:2424 (1977).

[33] C. W. Myles and J. D. Dow, Phys. Rev. B, 19:4939 (1979).

[34] Yu-Tang Shen and C. W. Myles, Phys. Rev. B, 30, 3283 (1984).

[35] A. Gonis. Green Functions for Ordered and Disordered Systems, volume 4 of Studies in Mathematical Physics. North Holland, Amsterdam, (1992).

[36] M. Jarrell and H. R. Krishnamurthy. Phys. Rev. B, 63:125102 (2001).

[37] R. J. Elliot, J. A. Krumhansl and P. L. Leath, Rev. Mod. Phys., 46, 3 (1974).

[38] A.H. Castro Neto, F. Guinea, N.M.R. Peres, K.S. Novoselov, A.K. Geim, Rev. Mod.Phys., 81, 109 (2009).

[39] J.S. Ardenghi, P.Bechthold, P.Jasen, E.Gonzalez and O.Nagel, Physica B, 427, 97-105 (2013).

[40] J.S. Ardenghi, P.Bechthold, E.Gonzalez, P.Jasen and A.Juan, Physica B, 433, 28-36 (2014).

[41] N. M. Peres, F. Guinea and H. Castro Neto, Phys. Rev. B, 73, 125411, (2006).

[42] I. S. Gradshtein and I. M. Ryzhik, Table of Integrals, Series, and Products, Ed. A. Jeffrey and D. Zwillinger, 7th edition, New York: Academic Press, (2007).

[43] T. M. Rusin, W. Zawadzki, J. Phys. A: Math. Theor., 44, 105201 (2011).

[44] Y. Ominato and M. Koshino, Solid State Commun., 51, 175-176, (2013).

[45] Y. V. Skrypnyk,V. M. Loktev, Phys.Rev.B, 82, 085436 (2010).

[46] G. Baym and L. P. Kadanoff, Phys.Rev., 124, 287 (1961).

[47] V. Janis, J. Kolorenc, Phys. Stat. Sol. (B), 241, 2032 (2004).

[48] V. Janis, V. Pokorny, Phys. Rev. B, 90, 045143 (2014).

[49] V. Janis, J. Kolorenc and V. Spicka, Eur. Phys. J. B., 35, 77-91 (2003).

[50] B. L. Altshuler, D. Khmel'nitzkii, A. I. Larkin, and P. A.Lee, Phys. Rev. B, 22, 5142 (1980).

[51] A. I. Larkin and D. E. Khmel'nitskii, Usp. Fiz. Nauk, 136, 536 (1982) [Sov. Phys. Usp. 25, 185 (1982)].

[52] G. Bergman, Phys. Rep., 107, 1 (1984).

[53] D. E. Khmel'nitskii, Physica B, 126, 235 (1984).

[54] H. Suzuura and T. Ando, Phys. Rev. Lett., 89, 266603, (2002).

[55] K. S. Novoselov, A. K. Geim, S. V. Morozov, D. Jiang, Y. Zhang,S. V. Dubonos, I. V. Grigorieva and A. A. Firsov, Science, 306, 666 (2004).

[56] Y. B. Zhang, J. P. Small, M. E. S. Amori and P. Kim, Phys. Rev. Lett., 94, 176803 (2005).

[57] C. N. R. Rao, A. K. Sood, K. S. Subrahmanyam and A. Govindaraj, Angew. Chem., 48, 7752 (2009).

[58] D. Wei and Y. Liu, Adv. Mater., 22, 3225 (2010). 
[59] H. Liu,Y. Liu and D. Zhua, J. Mater. Chem., 21, 3335-3345 (2011).

[60] T. O. Wehling, K. S. Novoselov, S. V. Morozov, E. E. Vdovin, M. I. Katsnelson, A. K. Geim and A. I. Lichtenstein, Nano Lett., 8, 1 (2008).

[61] W. Zhou, M. D. Kapetanakis, M. P. Prange, S. T. Pantelides, Phys. Rev. Lett., 109, 206803 (2012). 